\title{
Astrometry of Outer Solar System Bodies with Small Telescopes
}

\author{
J. Tichá and M. Tichý \\ Klět Observatory, Zátkovo nábřeži 4, CZ-37001 České Budëjovice, Czech \\ Republic
}

\begin{abstract}
Astrometry for an accurate orbit determination of distant objects is usually made with large telescopes ranging from 2 to 10 meters. In selected cases the bright(er) part of their population has been effectively observed with small 0.6 to 1 meter class telescopes. We point out here methods and techniques used at the Klet Observatory including our results. The extension of this programme using a new larger telescope is in preparation. We also mention a possibility of using some web tools for call for follow-up.
\end{abstract}

\section{Astrometry of distant objects}

We have started the Klet Observatory distant objects astrometric observations with recovery of Centaur $1997 \mathrm{CU}_{26}$ and follow-up astrometry of the scattered disk object $1996 \mathrm{TL}_{66}$ in 1997 (Marsden 1997). We use a 0.57-m f/5.2 reflector equipped with a CCD camera SBIG ST-8 and Klet software package for two different techniques for a detection of slowly moving objects fainter than $\mathrm{V}=20^{\mathrm{m}}$ - single images or co-added images.

\section{Astrometry using single images}

These methods are usually used for objects brighter than $\mathrm{V}=20.5^{m}$. We use single 120 or 180 -second exposures. There are several notable examples. Centaur $1999 \mathrm{UG}_{5}$ was discovered by the Catalina Sky Survey on 1999 Oct. 29. It was listed on the web NEO Confirmation Page (the NEOCP) established by the Minor Planet Center to call for confirmatory and follow-up astrometric observations of newly discovered unusual objects. We found it near the prediction and made independent confirmatory observations on 1999 Oct. 29.82 UT, when the total daily motion of this object was 0.09 degrees and its brightness was $\mathrm{V}=18.7^{\mathrm{m}}$. Following astrometric observations and orbit computation confirmed the Centaur character of $1999 \mathrm{UG}_{5}$ orbit (Marsden 1999).

Consequently we selected $1999 \mathrm{UG}_{5}$ as the target for recovery in August 2000 , when it appeared at the western elongation of about 90 degrees. The recovery of $1999 \mathrm{UG}_{5}$ was made at Klet on 2000 August 25.02 UT. It confirmed an independent recovery made at Linz (Marsden 2000). Its brightness was $\mathrm{V}=19.6^{m}$ and it was found 26 arcseconds from the predicted position. 
Distant object $1999 \mathrm{TD}_{10}$ was discovered by Spacewatch on 1999 Oct. 3 and also listed on the NEOCP. We obtained its positions on 1999 Oct. 7 UT, when the total daily motion of this object was 0.05 degrees and its brightness was $\mathrm{V}=19.0^{\mathrm{m}}$.

Following astrometric observations, including observations obtained at Klě́ on 1999 Nov. 29 after 30 days delay from previous observations, resulted in orbit computation suggesting a scattered-disk orbit (Williams 2000). The method of single images was also used for astrometry of the transneptunian $2000 \mathrm{~EB}_{173}$. We obtained two positions on 2000 June 3.90 UT and one on June 7.89 UT, both using 120 second exposures. The total daily motion was 40 arcseconds and its brightness was about $\mathrm{V}=20^{\mathrm{m}}$. All residuals are smaller than 2" (Tichá\& Tichý 2000).

\section{Astrometry using co-added images}

The method of co-added images is used for astrometry of objects fainter than $\mathrm{V}=20.5^{m}$. We take a dozen of exposures from 60 to 120 seconds and then we co-add these images. The limiting factor for co-adding is the motion of the target object and used pixel size. The limiting magnitude for such co-added images is near $\mathrm{V}=21^{m}$. We obtained positions of TNO $2000 \mathrm{GN}_{171}$ on 2000 June 18.87 UT and on June 19.87 UT, both using eleven co-added 60-seconds exposures. The total daily motion was 13 arcseconds and its brightness was about $\mathrm{V}=20.6^{m}$. All residuals are smaller than 2" (Tichá \& Tichý 2000).

\section{The new project KLENOT}

We decided to extend the Klet minor planet programme to fainter objects using a new 1-m reflector equipped with a more efficient CCD camera. The KLENOT goal in the outer Solar System is to contribute to the accurate orbit determination of distant objects. We plan to perform follow-up astrometry and recoveries of Centaurs and brighter transneptunians up to limiting magnitude $\mathrm{V}=22^{m}$.

Acknowledgments. This work has been supported by the Grant Agency of the Czech Republic Reg. No. 205/98/0266.

\section{References}

Marsden, B. G. 1997, Minor Planet Electronic Circular 1997-C12, 1997-S14

Marsden, B. G. 1999, Minor Planet Electronic Circular 1999-V09

Marsden, B. G. 2000, Minor Planet Electronic Circular 2000-Q21

Tichá, J., Tichý, M. 2000, Minor Planet Circulars 40747, 40916

Williams, G. V. 2000, Minor Planet Electronic Circular 2000-N23 